\title{
Regional models for distributed flash-flood nowcasting: towards an estimation of potential impacts and damages.
}

\author{
Guillaume Le Bihan ${ }^{1}$, Olivier Payrastre ${ }^{1, a}$, Eric Gaume ${ }^{1}$, David Moncoulon ${ }^{2}$ and Frédéric Pons ${ }^{3}$ \\ ${ }^{1}$ LUNAM Univeristé, Ifsttar, Département GERS, Lab. Eau Environnement, Route de Bouaye, CS4, 44844 Bouguenais cedex, France \\ ${ }^{2}$ CCR, 157 boulevard Haussmann 75008 Paris, France \\ ${ }^{3}$ CEREMA DTer Méditerranée, Pôle d'Activités Les Milles, Avenue Albert Einstein, CS 70499 Aix en Provence Cedex 3, France
}

\begin{abstract}
Flash floods monitoring systems developed up to now generally enable a real-time assessment of the potential flash-floods magnitudes based on highly distributed hydrological models and weather radar records. The approach presented here aims to go one step ahead by offering a direct assessment of the potential impacts of flash floods on inhabited areas. This approach is based on an a priori analysis of the considered area in order (1) to evaluate based on a semi-automatic hydraulic approach (Cartino method) the potentially flooded areas for different discharge levels, and (2) to identify the associated buildings and/or population at risk based on geographic databases. This preliminary analysis enables to build a simplified impact model (discharge-impact curve) for each river reach, which can be used to directly estimate the importance of potentially affected assets based on the outputs of a distributed rainfall-runoff model. This article presents a first case study conducted in the Gard region (south eastern France). The first validation results are presented in terms of (1) accuracy of the delineation of the flooded areas estimated based on the Cartino method and using a high resolution DTM, and (2) relevance and usefulness of the impact model obtained. The impacts estimated at the event scale will now be evaluated in a near future based on insurance claim data provided by CCR (Caisse Centrale de Réassurrance).
\end{abstract}

\section{Introduction}

Hydro-meteorological forecasting is an essential component of real-time flood management. It provides crucial information to crisis managers to anticipate, locate and quantify the floods which will hit the areas at risk. In the particular case of flash floods which may affect watersheds of limited extent spread over the territory, suitable forecasting systems are still currently under development over the world. The first proposed methods were focussed at gauged stream sections where rainfallrunoff models could be calibrated [1,2]. The most recent developments aim at providing forecasts also at ungauged locations and often rely on highly distributed hydrological models and on radar based QPEs or rainfall nowcasts as input information [3, 4, 5, 6, 7]. Such models provide indications of possible flood magnitudes, but are still rarely designed to directly account for the possible associated impacts which is the information needed by the crisis managers in real time to take appropriate decisions such as allocation of rescue means. The translation of flood magnitude into local impacts requires, indeed, a detailed knowledge of the flood extent related to the forecasted discharges as well as the exposure and vulnerability of the considered areas. This may be well known at a very local scale (local authorities, inhabitants of flood prone areas,..), but much more difficult to assess

\footnotetext{
a Corresponding author: olivier.payrastre@ifsttar.fr
}

and incorporate at the much larger scale at which hydrometeorological forecasting systems are generally implemented. Considering that these systems are designed to monitor a very large number of small rivers spread over large territories, a large number of simultaneous alarms may be generated in case of a significant rainfall event. Thus, providing directly information on local vulnerabilities and associated possible impacts would probably be helpful for coordination managers to have a faster evaluation of the situation and to focus their actions on the most problematic situations.

The approach developed in this paper aims at a direct evaluation of flash flood impacts on inhabited areas based on a complete hydro-meteorological, hydraulic and impact assessment simulation chain. The question of flash flood impact prediction has already been addressed in some previous works which were up to now mainly focused on road inundation risks [5]. The methodology developed herein is based on a comprehensive analysis of the study area in order to build an impact model for each river reach incorporated in the hydro-meteorological simulation chain. This analysis is based on simplified 1-D hydraulic simulations to evaluate the extent of the flooded areas for different discharge levels. Land use databases are then used to evaluate the number of 
buildings in the estimated flooded area. Based on these precomputed values, a relation between the discharge and the amount of affected buildings is adjusted for each considered river reach to be used for the real-time forecasts.

Even if the approach developed may appear relatively straightforward, its application on a very detailed stream network including small watersheds prone to flash floods, may be too complex to enable an application at a large scale (more than $100.000 \mathrm{~km}^{2}$ ). Therefore, the challenge has been here to define a simplified and automatic procedure for the elaboration of the impact model, with the objective to limit its implementation time to a reasonable level and to limit also the needs for manual corrections. This objective of simplification has been considered as a priority, placed before the accuracy of the computation of flooded areas and associated possible impacts. In other words, a decrease of the quality of the impact model has been accepted to guaranty its applicability at a large scale including a very detailed stream network.

This article presents the proposed method and its application on a test case study. The accuracy of the impact model, is tested against data of recent floods having recently affected the considered area. The article is organised as follows: the first section presents the application case study and the datasets, the next section presents the methodology developed for the fast computation of flood extents and the associated impact model. This section also describes the procedure used for the evaluation the results. The results are presented in section 4 and discussed in section 5 , in which the future perspectives in terms of validation are also presented. Lastly, section 6 presents the conclusions of this work.

\section{Presentation of the case study}

\subsection{The region of Alès in the Cévennes area, south eastern France}

The study has been focused on an area of $1990 \mathrm{~km}^{2}$ including the town of Alès in south eastern France. This area is located in the core of the Cévennes region, well known to be prone to frequent and intense flash floods. Moreover, this area has been identified during the implementation of the EU flood directive as one of the areas the most at risk over the whole French territory. For this reason, it was included in the list of Areas with Potential Significant Flood Risk (APSFR) selected for the application of the flood directive. This exposure is mainly related to the presence of the town of Alès, but also to other highly vulnerable smaller towns such as Anduze (see figure1).

This region is shown on figure 1, indicating the exact limits of the APSFR of Alès. This territory is part of two main watersheds: the Gardon d'Alès and the Cèze rivers. These two main rivers have their upstream course in the Cévennes reliefs, and reach in their downstream part a plateau area with limited slopes. The APSFR of Alès is located just in the transition zone between the mountainous and plateau areas. Therefore, this case study includes a large variety of river bed configurations including steep and narrow v-shaped valleys, up to flat and wide floodplains. Some statistics about the river bed characteristics are provided in table 1 .

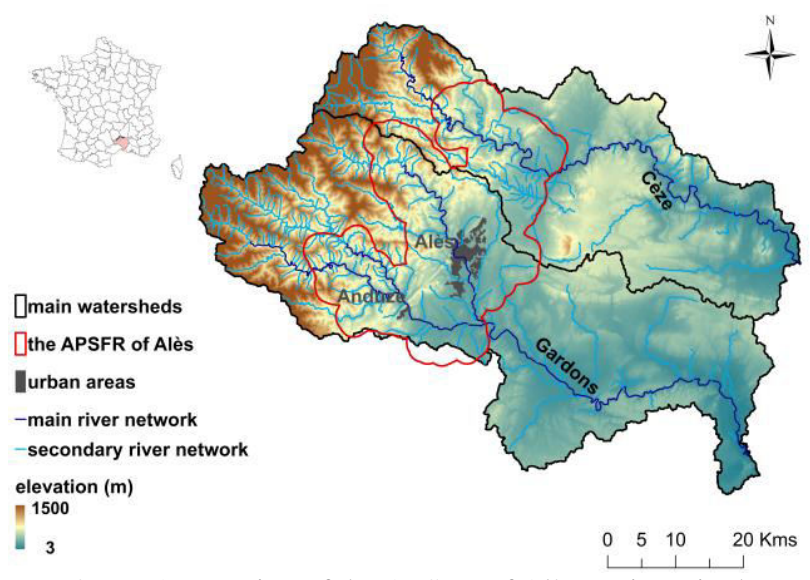

Figure 1. Location of the APSFR of Alès territory in the Gardon and Cèze watersheds.

One part of the river network in this area is already covered by the French national flood forecasting system. However, significant damages associated to flash floods also frequently occur on upstream rivers which are currently not monitored. A detailed analysis of the features of the river network, based on a DTM treatment, shows that the selected area includes $400 \mathrm{~km}$ of river streams having at least $5 \mathrm{~km}^{2}$ upstream catchment surfaces, divided in 192 river reaches (a river reach being defined as the portion of river located between two confluences), among which only 70 reaches $(132 \mathrm{~km})$ are covered by the current flood forecasting system (see main network on figure 2). This illustrates both, the relevance

\begin{tabular}{|c|c|c|c|c|c|c|}
\hline \multirow{2}{*}{ Location } & \multicolumn{2}{|c|}{ Bed slope $(\%)$} & \multicolumn{2}{|c|}{ River bed width (m) } & \multicolumn{2}{|c|}{ Floodplain width (m) } \\
\hline & average & $\min -\max$ & average & $\min -\max$ & average & $\min -\max$ \\
\hline Main network & 0.48 & $0.22-1$ & 37 & $16-84$ & 470 & $120-1670$ \\
\hline Secondary network & 3.41 & $0.17-20$ & 7 & $2-34$ & 430 & $60-3130$ \\
\hline
\end{tabular}

Table 1. Characteristics of the river network considered in the case study (extracted from SYRAH database). 
and the difficulty associated with the implementation of complementary flash flood forecasting systems on the very dense stream networks prone to flash floods. Even if the extent of the case study considered here is not very large, the number of rivers reaches to be integrated in the models is significant and may cause difficulties associated to the lack of time available to implement and test the models. It is noteworthy that an elementary catchment surface of $5 \mathrm{~km}^{2}$ has been selected here, and that decreasing this surface would lead to highly increase the quantity of river courses to be considered. This finally illustrates the importance of defining very simple and efficient approaches for the implementation of flash flood forecasting, as far as the objective is to apply such approaches in areas of relatively large extent (largely exceeding the extent of the case study considered herein). This point is particularly important herein since the main objective is the definition of a flash flood impact model based on hydraulic simulations, which may represented a much time consuming task when implemented over large areas.

\subsection{Available data}

\subsubsection{Digital Terrain Model}

The implementation of the impacts model has been based on a $20 \mathrm{~m}$ resolution DTM produced in 2007, which altimetric accuracy is less than $20 \mathrm{~cm}$ in no-vegetated areas, and less than $1 \mathrm{~m}$ in vegetated areas. This DTM was interpolated at a $5 \mathrm{~m}$ resolution for the purpose of the study. Therefore, the quality of the terrain information used herein remains limited: the use of a real $5 \mathrm{~m}$ resolution DTM from Lidar measurements would probably lead to results of better accuracy.
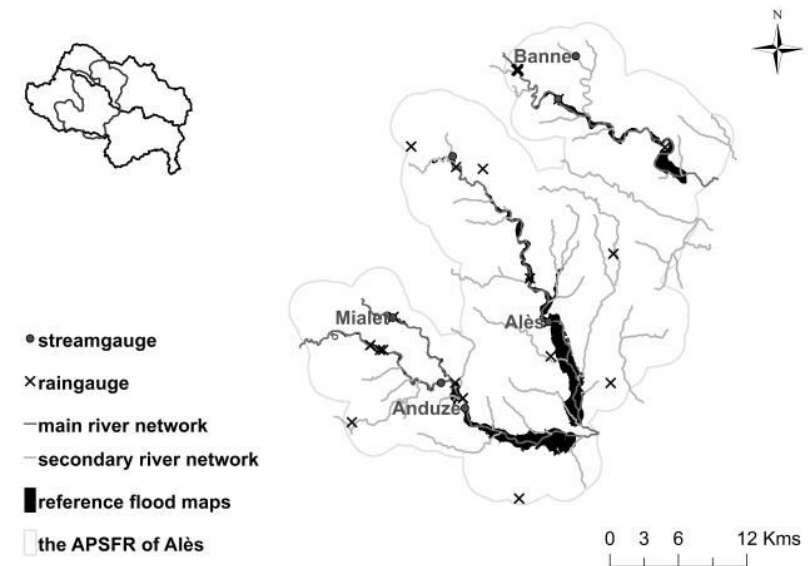

Figure 2. River network considered in the APSFR of Alès (5 $\mathrm{km}^{2}$ upstream catchment surface), coverage of reference flood maps and position of available stream gauges.

\subsubsection{Rainfall and discharges}

The region is equipped with relatively dense stream gauge and rain gauge networks, complemented with three weather radars. The positions of stream gauges are presented on figure 2. Thanks to the OHMCV observatory, this data is carefully checked and can be considered as exceeding conventional quality standards. However, given the limited possibilities to make direct flow measurements during intense flash floods, the rating curves are often extrapolated, with consequently a large decrease of the quality of estimated discharges for high water levels and large floods.

\subsubsection{Flood quantiles}

The regional SHYREG database, providing flood quantiles of different return periods (from 2 to 1000 years) on the whole river network, has been used herein to assess the discharge quantiles used to build the impact models for each river reach. This database relies on the combined regionalization of parameters of a rainfall stochastic generator and of a distributed hydrological model [8]. It has to be noticed here that both the work of regionalization and some specificities of watersheds such as karts and urban areas, snowmelt, or hydraulic structures (dams) have not been considered in the SHYREG approach and may locally reduce the reliability of SHYREG data.

However, the accuracy of information on flow frequency is not crucial herein for the implementation of the impact model: it just enables to derive flood maps for discharges values of relatively homogeneous magnitude in all the considered area.

\subsubsection{Reference flood maps}

Thanks to the recent application of the EU flood directive in the considered area, a great effort was put on flood extent mapping, enabling to produce detailed maps of probable flood extent for three reference events: a common event ( 30 year return period), a medium event (300 year return period), and a large event (exceeding a 1000 year return period). Therefore, 3 reference flood maps are available for some of the considered river reaches. These maps were obtained based on a 1-D hydraulic modelling conducted by an expert in hydraulics. The results were carefully validated using all the available information (including the extent of the exceptional september 2002 flood). These maps will be used for the validation of the flood areas estimated for the purpose of the flash flood impact model implementation. Unfortunately these maps, presented on figure 2, were produced on one part only of the river network considered herein which limits the validation possibilities: the river network covered represents $192 \mathrm{~km}$ (out of $400 \mathrm{~km}$ included in the case study) and includes 84 river reaches (out of 192).

\section{Methodology}


a)

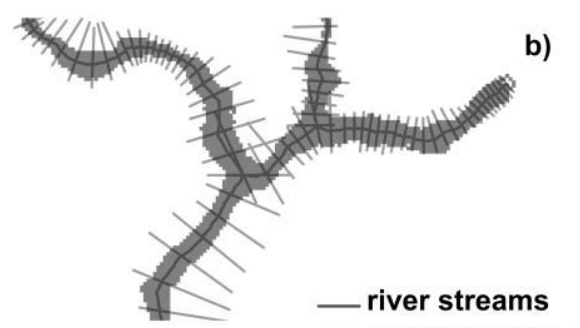

approximated profiles position c)

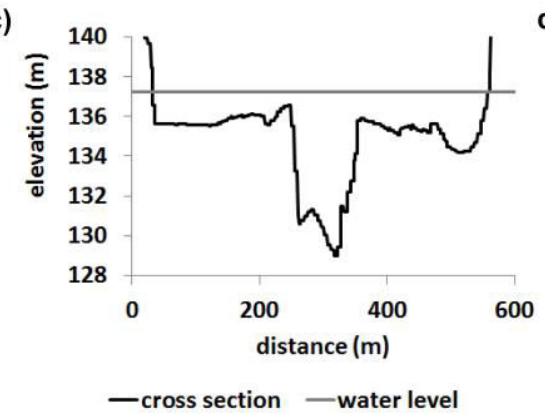

d)

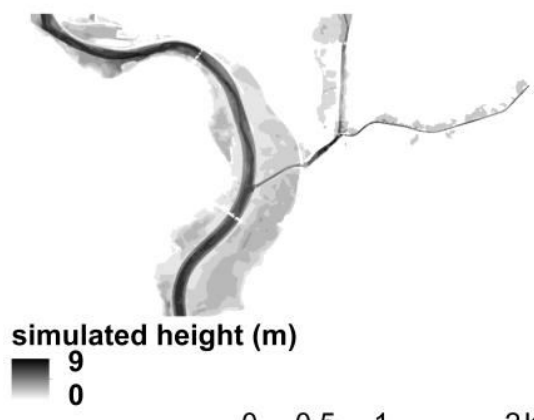

$\begin{array}{llll}0 & 0,5 & 1 & 2 \mathrm{Kms}\end{array}$

Figure 3. Overall principle of the computation of flood maps based on CartinoPC software: a) input information (position of river streams and approximate extent of flood area), b) position of profiles for the 1-D hydraulic model, c) computation of water levels (1-

D hydraulic model), c) map of flooded areas and water depths obtained after post-treatment..

As indicated in the introduction, the approach developed herein is based on a pre-evaluation of the extent of flooded areas for different discharge levels. This evaluation should be obtained in an as simple and automatic as possible way in order to enable its implementation on a large number of river reaches. Therefore, it has been based on a 1D hydraulic model run in steady state mode, and implemented automatically based on a high resolution DTM. This work has been based on an adapted version of the Cartino software [9], enabling to build the structure and run the model in a iterative way until satisfactory results are obtained. The Cartino parameters have been adapted to the objectives of this study, and some additional post treatments have been added to remove the most identifiable inconsistencies in the outputs.

The whole procedure is presented hereafter in the following way: first, the overall principles of the Cartino software are presented; then the implementation details (parameters, etc) are provided; the additional posttreatments introduced are presented; lastly, a short discussion about the main remaining sources of errors is proposed.

\subsection{The Cartino software}

The Cartino software enables to build and run automatically a 1-D hydraulic model on the considered river network to finally obtain a simplified evaluation of potential flooded areas for selected discharge values (discharges may be varied for each stream reach but all computation are performed in steady state mode). The computation is achieved in 3 main steps presented in figure 3: 1 - a pre-treatment module is used for positioning the cross sections, 2 - a module prepares the input files (cross sections shapes) and runs a 1-D hydraulic model (Mascaret or Flutor) for the computation of the longitudinal water level profilefor the considered discharge values, and 3 - a post treatment intersects the profiles and the DTM to estimate the flood extents and the associated spatial distributions of water depths.

To ensure a high level of automatization, important simplifications are introduced in the structure of the hydraulic model used: cross section shapes are estimated based on a simple interpolation with the high resolution DTM (without additional topographic information); bathymetry is not considered; specific sections (weirs, bridges) are not represented; a unique roughness coefficient is used for all stream reaches, without distinction between main channels and floodplains. Of course all these simplifications have a large impact on the results, even if they are necessary to obtain the required level of automatization of computations: this point will be evaluated and discussed in the next sections.

The first two modules of Cartino are run in an iterative way to optimize commonly the couple width of cross sections distances between cross-sections, with the objective to finally ensure that all cross sections are wide enough for the considered discharge and that no overlapping is present between two successive cross sections. This procedure is initiated based on the position of the river reach and a first possible extent of the flooded area (provided as input) which is used to estimate a default width of each cross section. The associated distances between profiles are then defined as a proportion of each profile widths (proportion to be defined as input parameter). After each run, the width of 
each profile is checked according to the computed water elevation, and it is enlarged if necessary in a proportion defined as input parameter. Distances between profiles are adapted in consequence. The roughness to be used coefficient is also defined as an input parameter $(n=0,05$ was used herein).

The choice of the input information (default extent of flooded area, parameters values for distance between cross sections and increase of cross sections widths) has also a great impact on the results and on the computation times. For instance, a too large default flooded area or a too fast increase of cross sections widths may lead to incorporate in the model some depressions located nearby the river bed, but not connected to it for the considered discharge (see figure 4): this leads both to simulate flooded areas of too large extent at the considered profiles, and to decrease the elevation of the simulated water level, with a repercussion on the simulations results at the upstream profiles (possible under estimation of water level). On the other hand, a too narrow initial flooded area and/or too slow increase of profile widths will highly increase the computation times.

To cope with these difficulties, the software offers the opportunity to change the position and/or modify the widths of any profile after a first iterative run.

\subsection{Specificity of application of Cartino for the purposes of the study}

For the purpose of building a comprehensive impact model on the detailed river network considered in the case study, the objective is here to obtain a catalogue of flooded areas corresponding to different discharge quantiles. A wide range of quantiles has been selected to represent the entire panel of possible flood magnitudes: based on the SHYREG database, discharges corresponding to return periods of $2,5,10,20,50,100$, 200, 500, 1000 years have been selected for each river reach. For a given return period, the flooded areas have been computed for all river reaches in the region.

Depending on the flood quantile, the simulated flooded areas may be limited to the river bed or extend up to the entire floodplain. One crucial aspect is to determine as accurately as possible the discharge level at which the inundation of the floodplain begins. For this purpose, the Cartino software was applied in the following way:

- application first for the smallest discharge values (first run for $\mathrm{T}=2$ years), using a narrow flooded area extent as input and a progressive increase in cross sections widths to be able to estimate as accurately as possible the limits of the river bed.

- application to the next discharge quantile based on the results obtained for the immediately lower discharge as initial extent of flooded area.

A careful check of the results after each computation also enables to eliminate some errors related to wrong estimations of the shapes of some cross sections, mainly associated with the limits of DTM information used as input: bridges still appearing in the DTM, remaining noise due to dense vegetation. This verification was based on the comparison of the wetted area of the successive cross sections, with an automatic removal of cross sections appearing as inconsistent with the immediate downstream and upstream cross-sections.

\subsection{Additional post-treatments}

Despite the aforementioned precautions, some obvious inconsistencies still remain locally in the computed flooded areas: flooded areas disconnected from the river bed, some areas included in the estimated flood extent for a given discharge and not for the immediately larger one. Thus, a simple post-treatment was applied to the catalogue of flood maps to ensure a better overall consistency of the results. This enabled to:

- $\quad$ systematically remove disconnected flooded areas (see figure 4).

- $\quad$ systematically include each flooded area estimated in the one computed for the immediately larger discharge.
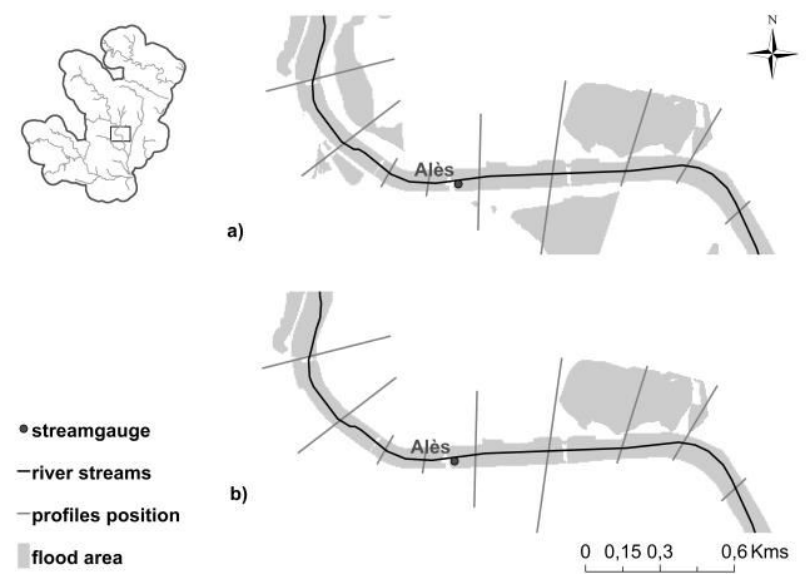

Figure 4. Illustration of additional post treatments applied to the Cartino results: a) initial Cartino results, b) after removal of disconnected areas.

\subsection{Remaining sources of uncertainty}

Although some precautions were taken to enhance the quality of the results, important limits and sources of errors remain, affecting the quality of the results. These remaining errors are mainly related to the objectives of the procedure applied herein, which was to guarantee a fast application and to accept the associated unavoidable errors. These errors are mainly due to:

- wrong representation of topography and characteristics of river bed due to the automatic definition of position of cross sections, and also to the limits of topographic information used (DTM),

- absence of representation of friction losses due to bridges and other singularities,

- choice of a fixed Manning roughness coefficient, equal to 0.05 ,

- absence of representation of dynamic effects due to the systematic computation in steady state mode. 
For these reasons, it is important to keep in mind that the information produced here cannot correspond exactly to the real extent of flooded areas. It represents a simplified estimation giving an order of magnitude of the level of flooding and enabling some comparisons at a regional scale.

\subsection{Evaluation of the results}

\subsubsection{Comparison with reference flooded areas}

The remaining error levels on flooded areas can be evaluated thanks to the availability of inundation maps produced for the application of the EU flood directive, which were used as a reference information (events of 30year and 300-year return period) .

To enable a direct comparison, intermediate layers were added to our flooded areas catalogue, using exactly the same flood discharges values as for the EU directive cartography (discharges of respectively 30 years and 300 years return periods). These results were then compared with the reference EU directive flooded areas on each river reach based on the following procedure: the common surface $(\mathrm{Sc})$ between estimated surface (ES) and reference surface (RS) was first considered (see figure 5), enabling to define the excess (Se) and default (Sd) surfaces as follows:

$$
\begin{aligned}
& E S=S c+S e \\
& R S=S c+S d
\end{aligned}
$$

A synthetic incoherent surfaces proportion (ISP) was then computed, representing the relative proportion of Se and Sd if compared to reference surface:

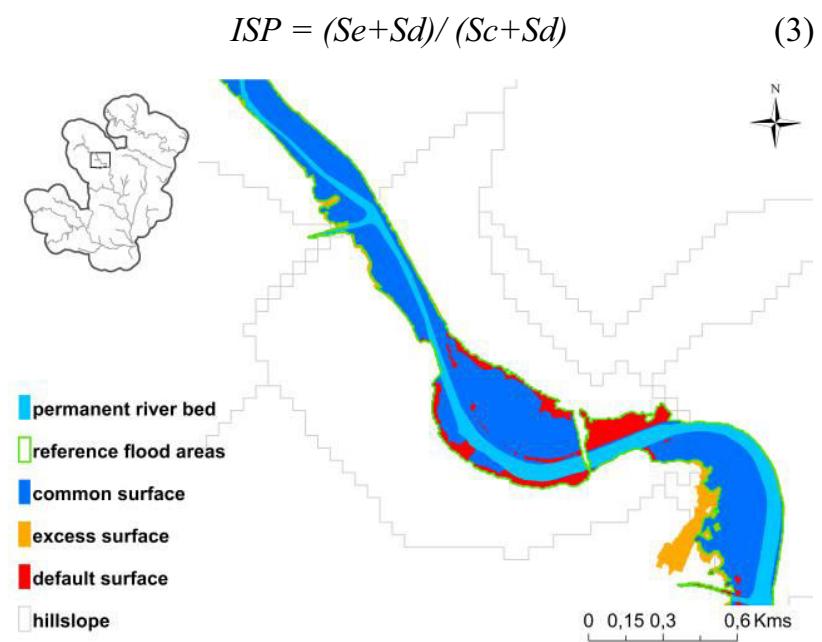

Figure 5. Illustration of comparison between estimated and reference inundated areas: definition of common surface $(\mathrm{Sc})$, excess surface (Se), and default surface ( $\mathrm{Sd})$.

It has to be noted that the portion of the river bed being permanently wet (represented on figure 5) may increase the value of Sc despite this area is not crucial for flood risks evaluation (absence of vulnerability). For this reason, this area was systematically removed from estimated (ES) and reference (RS) surfaces before the computation of Sc, Se, Sd, and ISPs. Therefore the evaluation proposed here is strictly limited to nonpermanently inundated areas.

\subsubsection{Comparison with rating curves established at stream gauges locations}

The presence of stream gauge stations with calibrated rating curves offers the opportunity to evaluate the accuracy of stage-discharge relation estimated by the model in the associated cross sections. This comparison was achieved after a careful selection of cross sections which better correspond to the location of the stream gauges. However, considering that the distances between cross sections are variable and may reach up to $100 \mathrm{~m}$, an additional cross section was added if necessary at the exact location of the gauging station.

a)

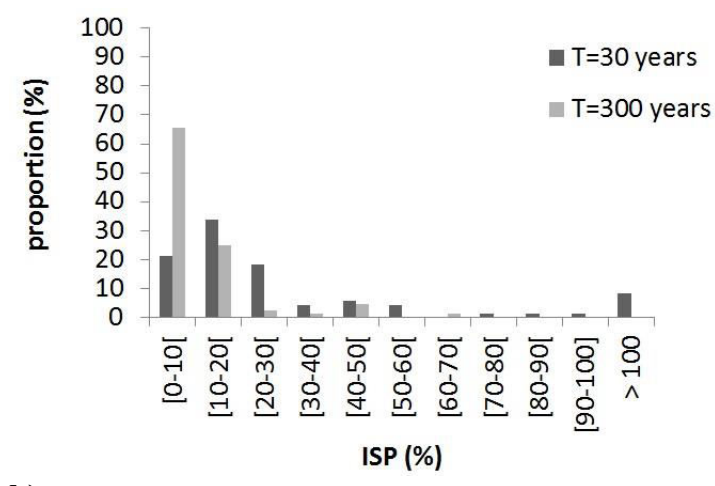

b)

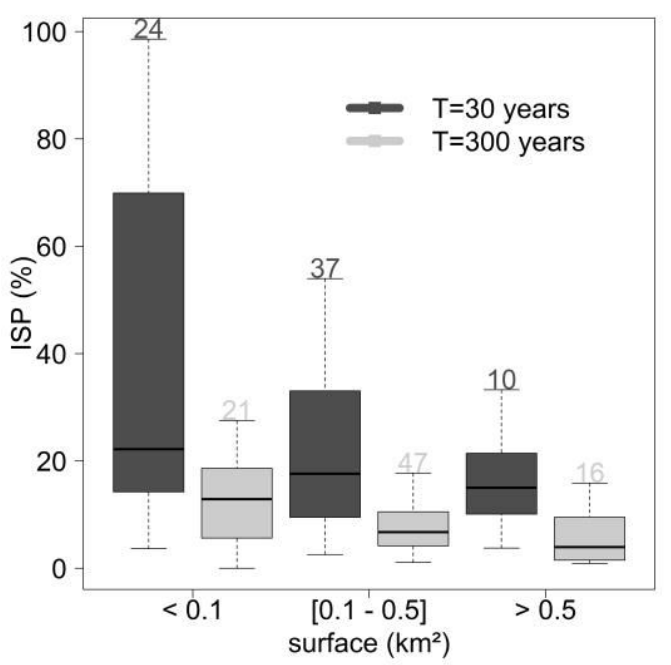

Figure 6. Synthesis of ISPs scores computed for river reaches on which reference inundation areas are available (71 reaches for the 30-year event, 84 reaches for the 300-year event): a) overall distributions, $b$ ) repartition in function of surfaces of reference flood areas(values above boxes indicate the associated number of reaches). 


\subsubsection{Comparison of impact models obtained}

The last evaluation step was based on the computation and comparison of impact models for each river reach. The impactmodels were computed here in a simple way by counting the number of buildings present in each estimated flooded area according to the IGN BDCarto database, to finally derive a continuous discharge-number of building relation. These impact models cannot be directly validated, but they were nevertheless compared to each other in order to verify whether the preliminary objective of the methodology developed herein - i.e. providing a hierarchy of possible impacts on a relatively large area - was achieved by obtaining significantly different impact estimations depending on the locations of the considered river reaches.

a)

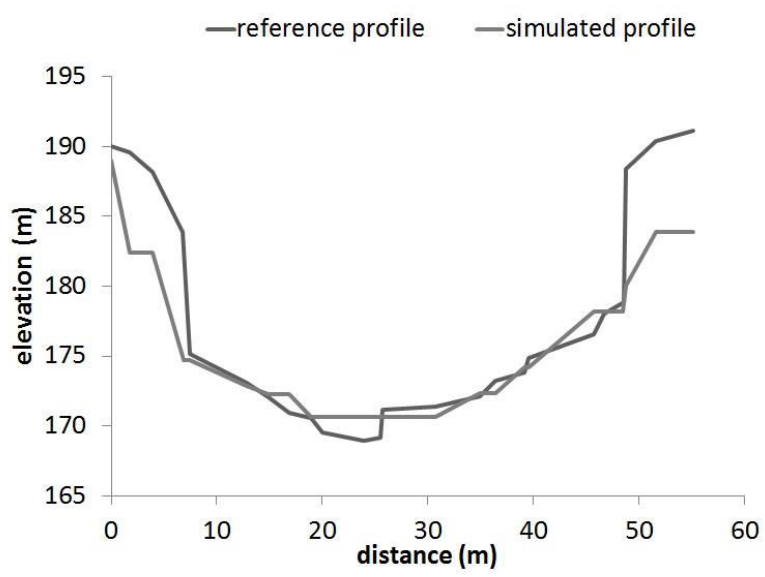

b)

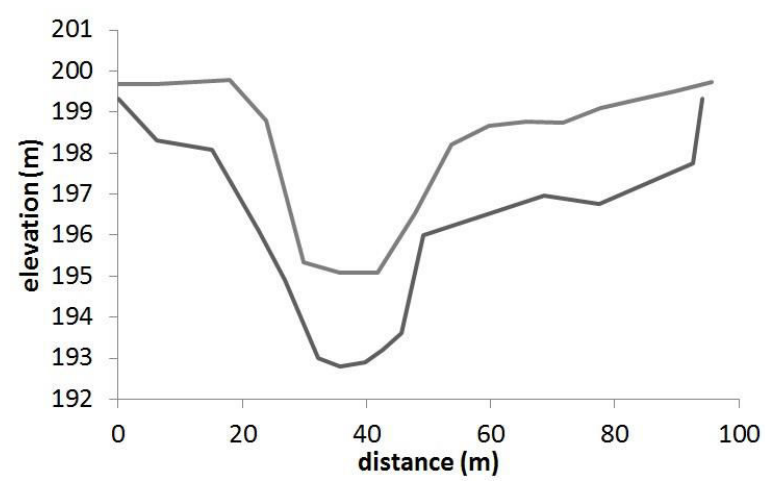

c)

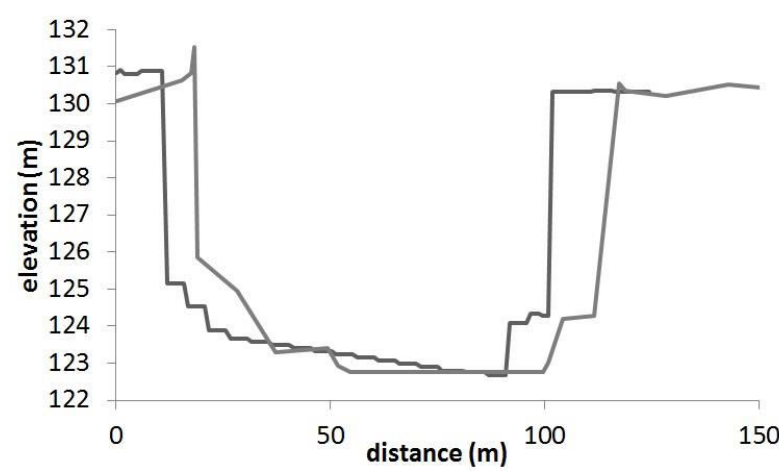

Figure 7. Examples of comparison of cross sections and water levels at three stream gauges:

a) Mialet station, b) Banne station, c) Alès station.

\section{Results}

\subsection{Extent of flooded areas}

Figure 6 presents the distributions of ISPs computed for the 84 rivers reaches on which the reference inundation maps are available ( 71 reaches for the 30 year event). This figure illustrates first the limited ISPs values obtained in a large majority of cases: ISPs rarely exceed $30 \%$. If we notice here that the ISP cumulates both default and excess areas, the real differences of surfaces of the estimated flood areas will be much more limited than the ISPs values: this suggests that the errors in the estimation of impacts will also be limited. These figures also illustrate significant differences depending on the magnitude of the simulated floods: the results obtained for the 300 year flood appear much more accurate, with ISPs rarely exceeding $50 \%$. This can be mainly attributed to the fact that the flood plains are almost
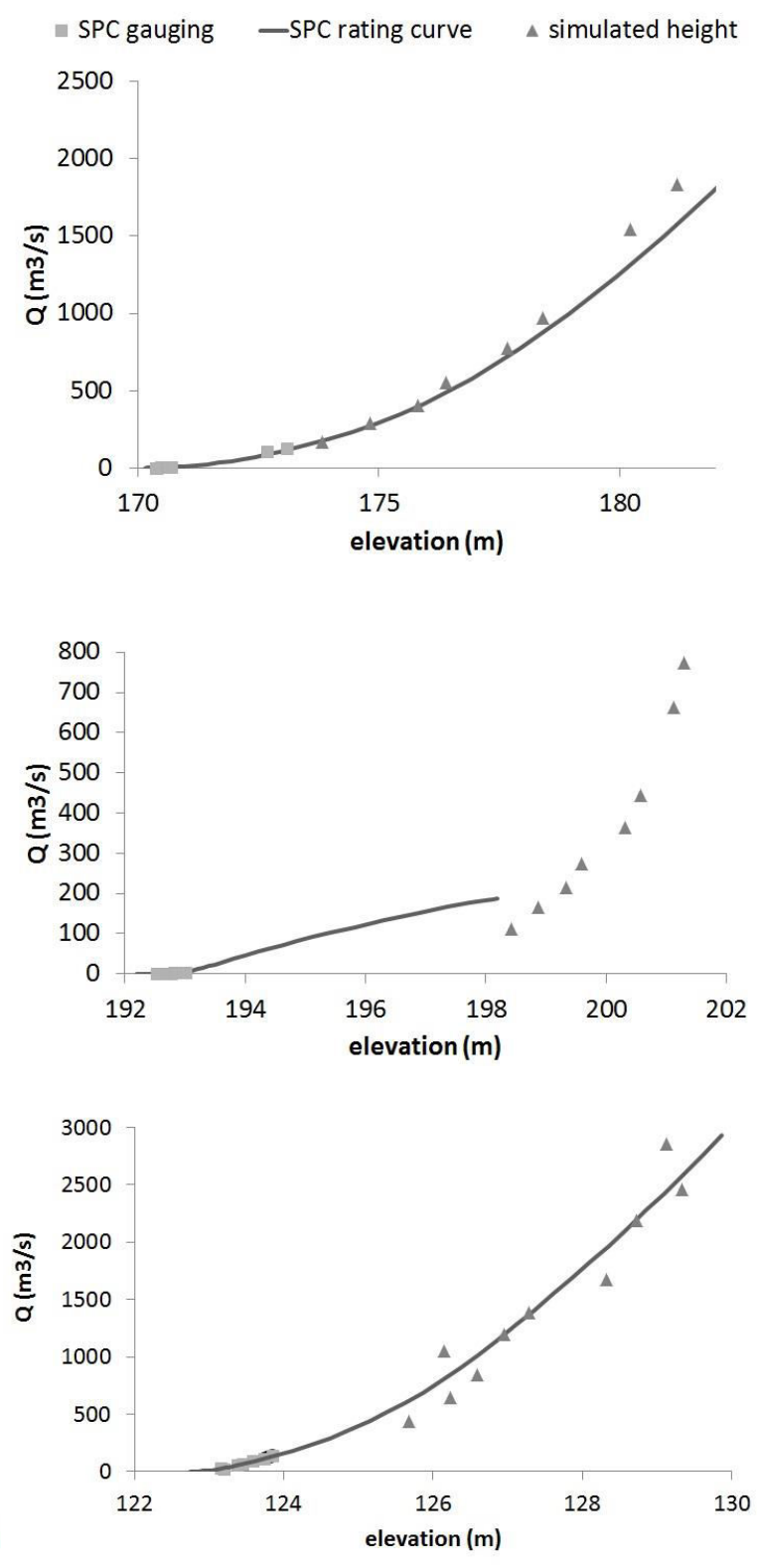
entirely flooded for this range of discharges, leading to limit the errors associated with the description of local flow conditions. On the other hand, the 30 year return period flood extent appears much more difficult to appraise, with a significant part of very large errors: ISPs exceeding $100 \%$ in almost $10 \%$ of the considered reaches. These large errors are mainly related to the crucial importance of the description of the characteristics of the river bed and of the flow conditions to correctly simulate the beginning of inundation: errors in crosssection widths or position, on roughness values, absence of description of head losses, will all result in large errors in this range of discharges. Therefore it appears completely logical that the simplified procedure used herein leads to results of significantly lower quality for moderate return period floods.

The analysis of repartition of ISPs depending on the surface of the reference flooded area (RS), provided on figure $6 \mathrm{~b}$, confirms the same tendency: ISPs are much more important for low RS values, illustrating the difficulty to appraise correctly the beginning of inundation. This tendency is also present for the 300 year flood but in a much limited extent: in this case, a limited value of the reference surface RS often implies a very deep and narrow river valley, highly limiting the risks of errors.

\subsection{Water levels at gauged river sections}

The results of comparison of stage discharge relationships are presented on figure 7 . This figure illustrates contrasted situations which are detailed hereafter.

The case of the Mialet station appears as an ideal situation, in which the shape of the cross section is very well represented in Cartino, and the water levels computed don't seem to be significantly affected by other sources of errors (choice of roughness coefficient, width of cross section, etc ..). This may be explained by the simple configuration of the valley (deep and relatively narrow), the limited presence of vegetation in the river bed, and the significant slope. A sensitivity test to the value of roughness coefficient can show in this case that the associated errors are of secondary order, provided that the cross section is well described.

The case of the Banne station is much more problematic since the topography of the river cross section is absolutely not well represented in the Cartino model. This may be attributed to local errors in the DTM used, maybe associated with the presence of dense vegetation. However, even if a significant error is made in the estimated elevation of the water level, this error will not necessarily be very problematic for the computation of inundated areas, since the same DTM including the same elevation bias will be used for this purpose. a)

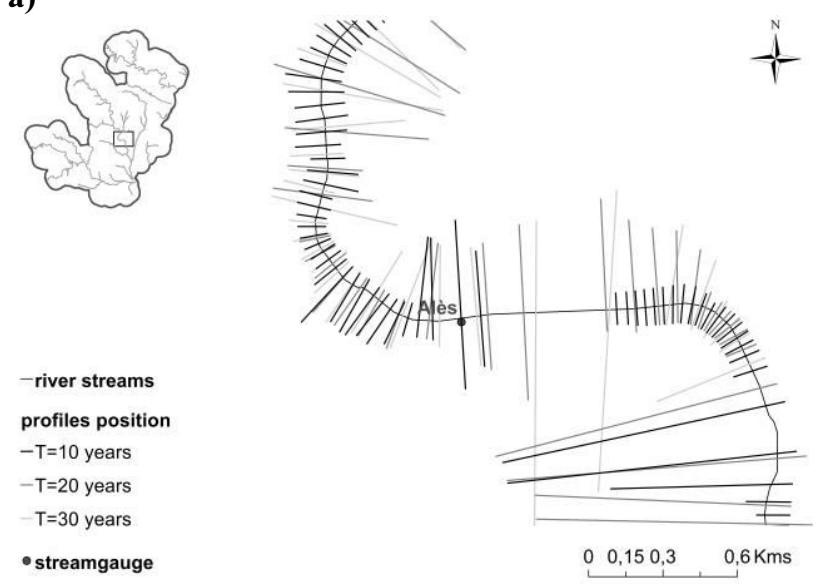

b)

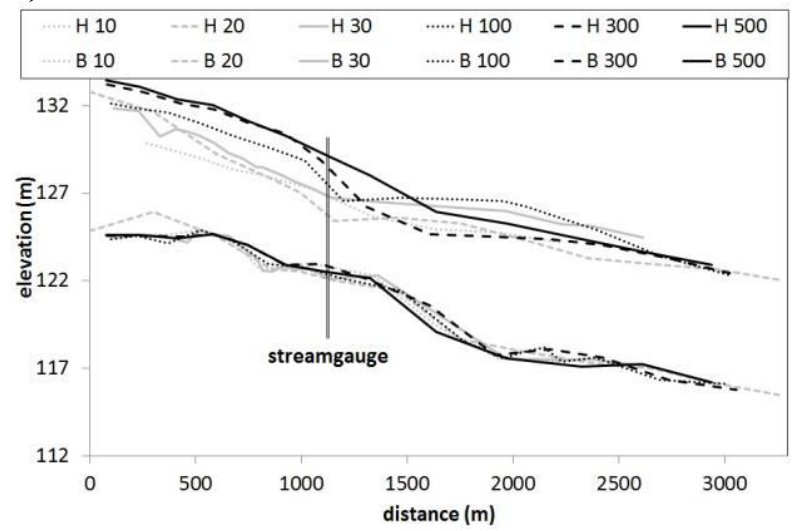

Figure 8. Illustration of the impact of cross sections widths on the simulated water levels nearby the Alès station: a) position of cross sections for different discharge levels, b) longitudinal profiles $(\mathrm{B})$ and computed water levels $(\mathrm{H})$.

The case of the Alès station is a more surprising one. Again in this case, the topography of the river bed appears well represented in Cartino. But the shape of the stage discharge relation appears very chaotic if compared to the rating curve of the station. A sudden decrease of water levels is simulated for discharge levels of 20 to 30 years return periods. The configuration of the river bed is different here from the previous cases. The section is located on the main Gardon d'Alès river, with a wide permanent river bed (about $100 \mathrm{~m}$ ), and a very flat floodplain including some large depressions. This represent a typical case in which the width of the profiles included in Cartino will have a great impact on the results. Figure 8 illustrates in detail what happens in this case: for limited discharge levels the cross sections defined by Cartino end at the riverbanks, leading to very consistent results; but for the $\mathrm{T}=20$ years discharge, a wide profile is incorporated in the hydraulic model, located just downstream the position of the station (see figure $8 \mathrm{a}$ ). As a consequence, a wide flooded area (disconnected from the riverbed, see figure 4) is considered by the 1-D model, leading to a significant decrease of simulated water levels in the considered section and also the upstream sections. In such a situation, the use of a 2-D hydraulic model could help in improving the delineation of the flooded areas but at the price of a dramatic increase of computation times. 


\subsection{Impact models}

The characteristics of the impact models obtained are summarized on figures 9 and 10. Figure 9 represents the geographical repartition of the maximum impact estimated at each river reach (number of buildings located in the 1000 year estimated flooded area). This figure confirms that the main urban areas, representing the main potential impacts are correctly identified by the model: a limited number of river reaches with very large estimated impacts. In addition, the impact model also provides an interesting hierarchy of the possible impacts in other locations: figure 10.a shows that the model identifies a large majority of river reaches for which the potential impacts are very limited, and therefore enables to discriminate the more limited number of river reaches for which significant impacts may be observed, even if remaining much more limited than in urban areas. It is likely that this kind of information will be of great help for the interpretation of forecasts provided in real time by distributed hydro-meteorological models.
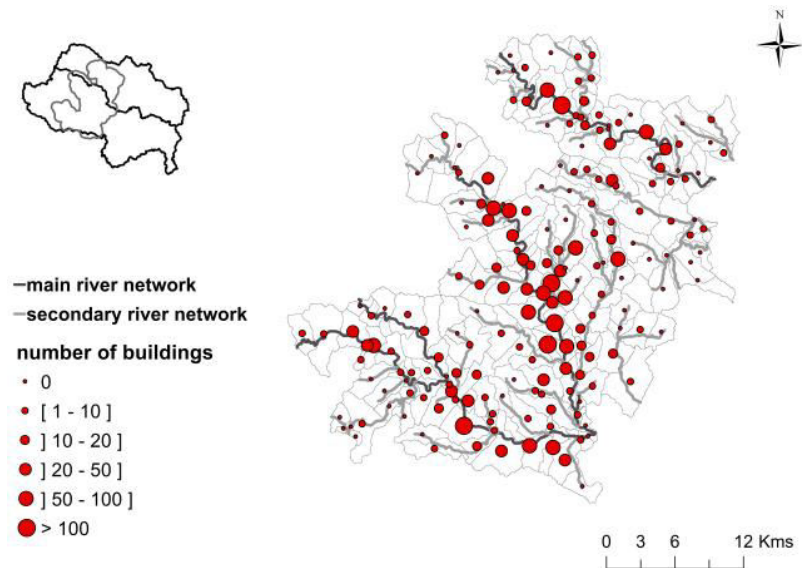

Figure 9. Map of the maximum impacts estimated for each river reach (number of buildings in the 1000-year flooded area).

Moreover, figure 10.b also shows that the impact model identifies an interesting hierarchy in the discharge return periods for which a significant part of the exposed assets begin to be affected. The model seems to be able to distinguish river reaches for which the inundation begins for relatively low discharges, or on the other hand for large discharges, providing interesting information about the flood levels (in terms of frequency) leading to the first potential damages. Of course, considering the relatively high uncertainty of estimated flooded areas in the beginning phase of the inundation, this information will probably be less accurate than the previous one (hierarchy of impacts for large discharges), and will probably include significant errors. But it is nevertheless an interesting information for the management of floods of intermediate magnitude, for which the location of damages do not necessarily follow the geographical repartition presented on figure 9 . a)

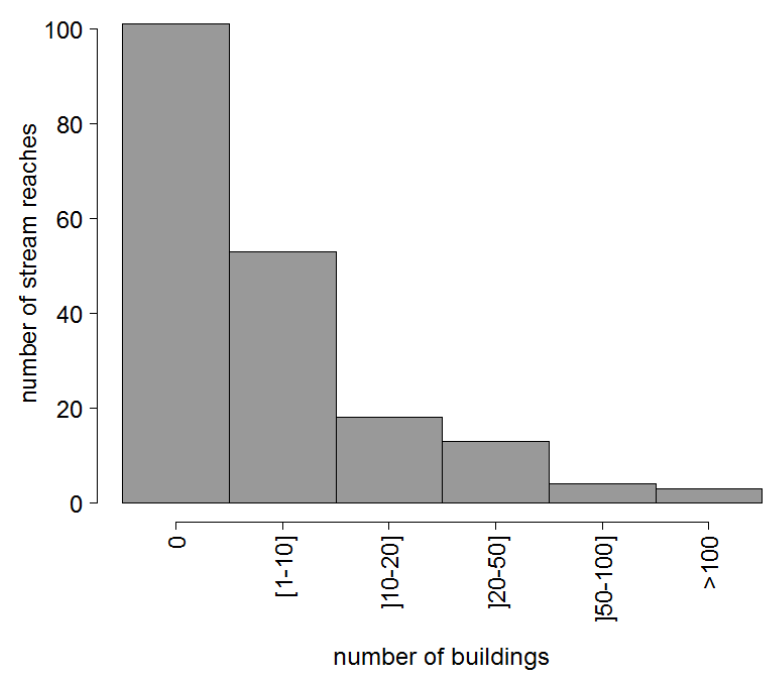

b)

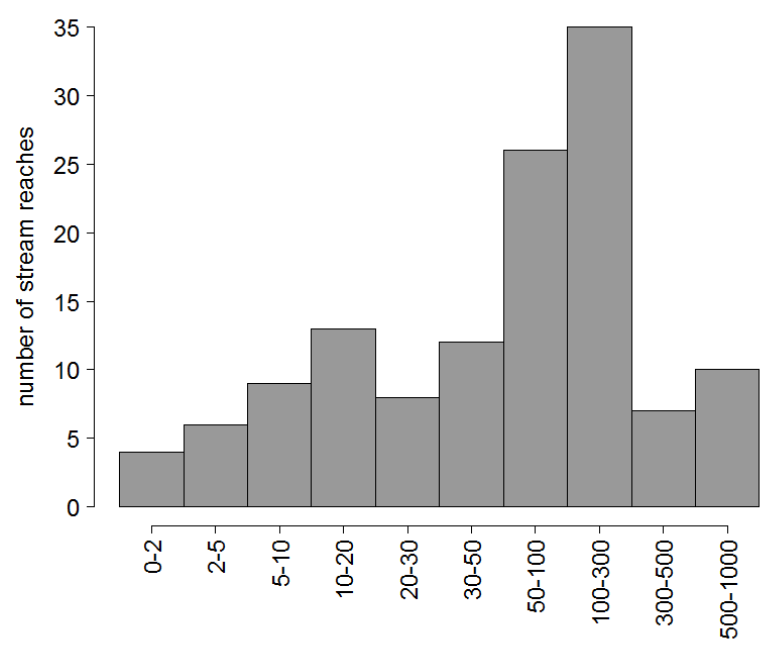

return periods

Figure 10. Main characteristics of the impact models obtained on the 192 considered river reaches: a) distribution of maximum impacts (1000 year flooded area), and b) distribution of discharge return periods leading to $50 \%$ of the maximum impacts.

\section{Discussion and further validations based on the CCR database}

The first validation results presented herein are focused on the accuracy of estimated flooded areas. These results show that if very large errors may be observed in a limited number of cases (typically less than $10 \%$ of the river reaches) for which the results should still be improved, in a large majority of cases the errors associated with the important simplifications introduced in the computation procedure remain much more limited (ISPs not exceeding 20 to $30 \%$ ). Therefore the procedure applied herein combines the advantage of its computational efficiency, enabling to generate estimated flooded areas on a very detailed river network and for a wide range of discharge levels, and of acceptable error levels considering the objectives of this study: giving a 
first estimation of the magnitude and hierarchy of impacts at a large regional scale.

The features of the impact model show that it contains useful information about the location of the vulnerable settlements, and could probably usefully complement the outputs of a rainfall runoff model to support crisis management and rescue operations. However, the combination of this impact model with a rainfall-runoff model will introduce additional sources of errors such as: errors in discharge estimations, interpolation between two estimated flooded areas for the estimation of the associated impacts. The final accuracy and usefulness of information on possible impacts will highly depend on the combination of these sources of errors. Finally, it clearly appears that this kind of approach still needs further validation to estimate its real efficiency in a close to real world situation. For this purpose, one objective is now to valuate insurance claim data collated by the CCR within the framework of its reinsurance traities with its clients. This data being confidential, the study will be based on aggregated information provided by CCR. This information, indeed, may provide an interesting synthesis of the location of the main damages to settlements at the event scale [10], to be compared with the impacts estimated by our model.

This kind of validation at the event scale is currently in progress, but we present here some preliminary results showing the overall consistency between the nature and representativeness of information collated by CCR and our impact model. Figure 11 presents for instance the correspondence between number of buildings identified in the impact model and the number of insurance policies of individual houses included in the CCR database in 2014 (limited here to the well geocoded policies, at street number precision or at street center precision). This graph shows a good overall consistency of both information sources with a proportion policies/buildings of 50 to $80 \%$ depending the considered area (10-year or 50-year flooded area).

Figure 12 shows the proportion of insurance claims/policies computed on the whole observation period covered by the CCR database (1998-2013), within the flooded areas used for the definition of the impact model. This figure shows again a good consistency of information, with a decreasing proportion of claims/policies ratios as the return period of the considered flooded area increases. It can also be noted that the ratios are significantly higher within the identified floodplains than outside.

Based on these first results, the challenge is now to use the claim data for the validation at the event scale of the entire simulation chain including both hydrological rainfall runoff model and impacts model.

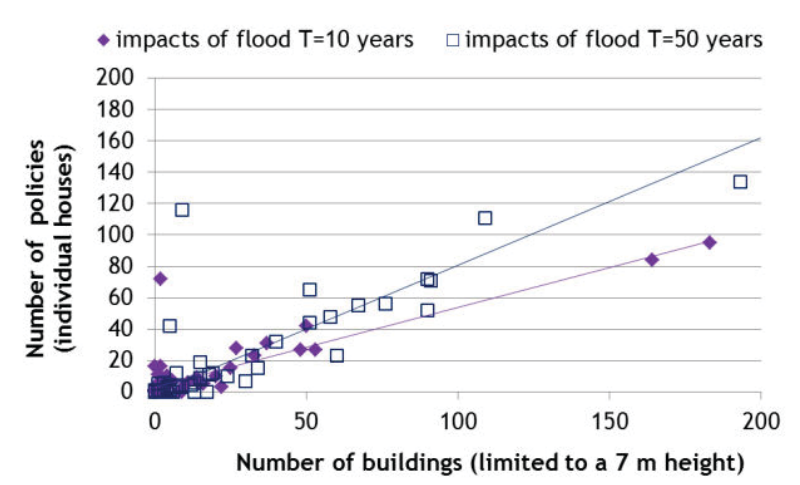

Figure 11. Comparison of the number of insurance policies included in the CCR claim database and of the number of buildings identified in the impact model within estimated flooded areas $(1$ point $=1$ river reach $)$.

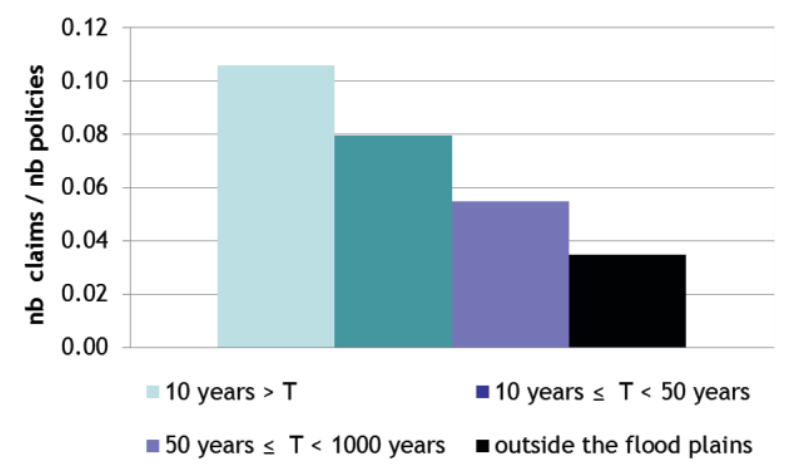

Figure 12. Ratios between number of claims and number of policies included in the CCR database within (and outside) estimated flooded areas.

\section{Conclusions}

The case study presented herein aimed to illustrate the potential benefits of a simplified impact model to complement the real time information provided by hydrological models for the monitoring and forecasting of flash floods. The impact model proposed is based on 1-D hydraulic computations achieved during a preanalysis phase: important assumptions are made, enabling to derive in a limited computation time a catalogue of inundation maps for different discharge quantiles.

The evaluation of this catalogue versus reference flooded areas shows that the level of errors, even if significant, remains acceptable for the monitoring and forecasting objectives. Moreover, this catalogue leads to a clear hierarchy of the exposure of the considered territory, including interesting information about the discharge levels above which the assets begin to be significantly affected.

Based on these promising results, the challenge is now to combine this impact model with a hydrological model to assess if the information on impacts obtained at the event scale can be valuable for crisis management operations. The validation of these results should be possible based on the insurance claim data collated by CCR. 


\section{References}

1. Georgakakos K.P. (2006). Analytical results for operational flash flood guidance. Journal of Hydrology, 317, 81-103.

2. Norbiato D., Borga M., Degli Esposti S., Gaume E., Anquetin S. (2008). Flash flood warning based on rainfall thresholds and soil moisture conditions: An assessment for gauged and ungauged basins Journal of Hydrology, 362, 274- 290.

3. Javelle P., Demargne J., Defrance D., Pansu J. and Arnaud P. (2014). Evaluating flash-flood warnings at ungauged locations using post-event surveys: a case study with the Aiga warning system. Hydrological sciences journal, 59(7), 1390-1402.

4. Gourley J.J., Flaming Z.L. Hong Y. and Howard K.W. (2014). Evaluating of past, present and future tools foe radar-based flash-flood prediction in the USA. Hydrological sciences journal, 59(7), 13771389.

5. Naulin J.P., Payrastre O. and Gaume E. (2013). Spatially distributed flood forecasting in flash flood prone areas Application to road network supervision in Southern France, Journal of Hydrology, 486, 8899.

6. Rozalis S., Morin E., Yair Y. and Price C. (2010). Flash flood prediction using uncalibrated hydrological model and radar rainfall data in a Mediterranean watershed under changing hydrological condition. Journal of hydrology, 394, 245-255.

7. Versini P-A., Berrenguer M., Corral D. and Sempere-Torres D. (2014). An operational flood warning system for poorly gauged basins : demonstration in the Guadalhorce basin (Spain). Natural Hazards, 71, 1355-1378.

8. Aubert Y. et al., (2014). The SHYREG flow methodapplication to 1605 basins in metropolitan France, Hydrological Sciences Journal, 59(5), 993-1005.

9. Pons F., Laroche C., Fourmigue P. et Alquier M. (2014). Flood hazard maps for extreme event scenario: the study of Nartuby river. La Houille Blanche, 2, 34-41.

10. Moncoulon D., Labat D., Ardon J., Onfroy T., Leblois E., Poulard C., Aji S., Rémy A., and Quantin A. (2014). Analysis of the French insurance market exposure to floods: a stochastic model combining river overflow and surface runoff, Nat. Hazards Earth Syst. Sci., 14, 2469-2485. 